\title{
A Topology Model with Energy- and Location-awareness based on the Laws of Internal Evolution of the Wireless Sensor Network
}

\author{
Dengchao Huang $^{1, a}$, Jing Fan ${ }^{1, b^{*}}$, Junhua Chen ${ }^{1, c}$
}

1 Key Laboratory of IOT Application Technology of Universities in Yunnan Province, Yunnan

Minzu University , Kunming, China ( 650500 )

ahuangdengchao19@163.com, banjing9476@163.com, ${ }^{\mathrm{c}}$ chenjunhuabj@163.com;

Keyword: complex network, location-aware, energy-aware, node distribution, attachment delete.

\begin{abstract}
In this paper, based on complex network theory, a topology model was proposed which is more accord with the nature of wireless sensor network. Actually, each node's actual location is crucial, in addition, the node energy consumption and communication distances between nodes are inseparable, so we tried to join location-aware mechanism into this wireless sensor network topology model with energy-aware mechanism. Besides, through theoretical analysis and simulation, we showed the influence on the network performance when the network have different node energy distribution and different node location distribution. Node energy distribution and node location distribution have a weak effect on the degree distribution $P(K)$, but which have much effect on the internal topological characterizations of the network. The node who has more energy will have more degrees to balance energy consumption, besides, when the node energy is more heterogeneous and node location is more centralized, the model showed that the network has higher clustering degree, and the network enjoys better performance in energy efficiency for transmitting data.
\end{abstract}

\section{Introduction}

In recent years, the complex network theory is applied to many fields of science, such as, the Internet, neural networks, social networks, electricity networks, network traffic, and so on. in 1999, Barabasi and Albert[1] proposed a scale-free network model. But in reality, the formation of network needs to go through much more complex steps. And if we want to simulate network, a variety of mechanisms is needed. Therefore, to ensure the ability to simulate a network with better effect, we need to consider much more practical factors, as well as detailed steps and methods.

Wireless sensor network (WSN) is composed of a large number of sensor nodes with working together. Wireless sensor networks have been applied to the fields of biological medicine, environmental monitoring, hazardous area remote control, and so on. Simulating the formation of wireless sensor networks is to provide a more reliable basis for the distribution of the location of nodes and setting energy of the nodes.

At present, many researchers have proposed many topology models for wireless sensor network based on the theory of the complex network model. Andras Farago [2] are reviewed many topology models, including all kinds of scale-free network model, and a lot of wireless sensor network model. Mahesar A W, Malik M etc. in [3] proposed application of calculus in the wireless sensor network model. Chen et al. [4] proposed an evolution local area world model for wireless sensor network based on classical BA scale-free model, which is robustness for random failure of the node. And this model provides a reference for constructing a robustness sensor network topology. Zhu [5] put forward two kinds of scale-free network model: energy perception (EAEM) evolution model and evolution model (EBEM), which are basic wireless sensor network evolution model. Zheng wang [6] propose two kinds of wireless sensor network topology structure: the linear growth model and accelerated evolution model, which are robustness in the situation random failure and deliberate attacks.

Presently, the research about the problem of the distance between node and node distributed 
model in complex network is few. Most of energy consumption is the process of node communication with others. And node energy consumption is increasing with the increase of the distance between nodes. And the spreading model of node will affect the distance between nodes in a certain extent. Our contributions are as follows: Firstly, in order to explore the effect on the properties of wireless sensor networks, which is caused by spreading model, we improve BA model [1] with adding location-aware mechanism. Secondly Combination of two important mechanisms of energy preferential attachment for link and node addition and energy antipreferential attachment for link and deletion contributes to investigating the complexity of WSNs. (3) the network degree distribution $P(K)$ can grow in the non scale state by the mean field theory analysis. The simulation of several critical topology characteristics are used to demonstrate the effectiveness of the proposed model in this paper.

The rest organization of this paper is as follows: in the second part, we propose a new wireless sensor network model. In the third section we analysis this kind of model with the mean field theory. Simulations experiments and discussion are given in section IV. Section V presents conclusion and the research point in the future.

\section{The Proposed Model for WSNS}

In this section, we propose a model for wireless sensor network based on scale-free networks. In the initial stage of this model, there are $m_{0}$ nodes and $e_{0}$ edges. The growth of the network is as follows:

(1)The Way of Node's Connection

Since a network with $m_{0}$ nodes, each step add a new node and connected to the existing $m$ nodes, one of them $\left(m \leq m_{0}\right)$.

(2)Preferential Attachment

The relationship between probability $\prod i$, which denote the probability that a new node is connected to an existing node $i$ and degree $k_{i}$ of node should satisfy formulation (2.1)

$\prod k_{i}=\frac{E_{i} * k_{i}^{*}\left(d_{i j}\right)^{(-1)}}{\sum_{j}^{k} E_{i} * k_{i}^{*}\left(d_{i j}\right)^{(-1)}}$

where $E_{i}$ is the residual energy of node $i ; k_{i}$ is the current node degrees and $d_{i j}$ is the distance between the node $i$ with the new node $j$.

Actually, each node's energy is limited in wireless sensor network. And the nodes with more energy means that the node has a stronger ability to connect to the new node. The node degree represent the number of edges of nodes and the ability of node to connect to others. Currently, few papers consider the actual distance between nodes in their models. The most of energy consumption is the process of node communication with others. And the actual communication distance is lager, the node consumes more energy. So add the actual distance parameters, that to make the whole network model is more practical.

(3)Links Deletion

Each step to probability $p(0 \leq p \leq 1), m^{*} p$ article existing edge will be deleted. $p$ denotes the deletion rate. Firstly, we will choose a node $i$ as deleted the edge of the end, and choose a node $j$, which is one of neighbor nodes of node $i$ as delete another endpoint, the selected probability of node $i$ is as follows:

$\prod * k_{i}=\frac{k_{i} *\left(E_{i}\right)^{(-1)} * d_{i j \max }}{\sum_{i} k_{i} *\left(E_{i}\right)^{(-1)} * d_{i j \max }}$ 
The selected probability of node $j$ is $\prod^{*} k_{j} / \sum_{j}^{o_{i}} \prod^{*} k_{j}$, then the edge between node $i$ and the node $j$ is deleted. Repeat this step $m^{*} p$ times. In the choice of deleting edge, low energy and high degree between nodes of the edges are more likely to be deleted. Actually, to prolong the life time of the entire network and achieve the whole network energy consumption balance, The node should delete some edges which connect with others, when the energy of the node is low. In the actual network, node energy consumption and the actual distance between nodes, these have exponential relation. In order to optimize the whole network, it should reduce energy consumption and to delete edge processing, the actual communication between nodes can be finished through multiple hops, rather than just to communicate over long distances by one hop.

\section{Network Analysis}

To present the entire network performance, in this section, it analysis how the energy distribution $\rho_{E}$ and the node distribution model can affect the node degree distribution $P(K)$, as well as the simulation the statistic parameters: the degree distribution $P(K)$, average clustering coefficient $C$, average path length $L$.

\subsection{Degree Distribution}

Degree distribution $P(K)$, which denote the probability that a randomly selected node has $k$ connection, is usually very important statistical character of large-scale complex network. In this paper, we use the mean field theory to analyze how $P(K)$ change, under the condition of different energy distribution models and different node distribution models.

The initial network have $m_{0}$ nodes, and has $e_{0}$ edge. And node $i$ has degrees $k_{i}(t)$ at time $t$. When the $t$ for sufficiently large, it can ignore initial $e_{0}$ edge in the network. When a new node is added to the system, let the node degrees $k_{i}(t)$ change over time:

$\frac{\partial k_{i}}{\partial t}=m \prod\left(k_{i}\right)-m p \prod * k_{i}\left[1+\sum_{j \in \text { lined }(i)} \prod * k_{j} / \sum_{j}^{o_{i}} \prod{ }^{*} k_{j}\right]$

In (3.1), when a new node add to the system, the degree of the node $i$ has changed .From the mean-field theory, we can get that: $\sum_{i} E_{i} k_{i} d_{i j}=N(t) * \bar{E} *\langle k(t)\rangle * \overline{d_{i j}}$, where $\bar{E}$ denotes the expected value of the node energy in the whole network; $N(t)$ denotes the number of node at time $t$; $\langle k(t)\rangle$ denotes the expected value of the node degree in the whole network; $\overline{d_{i j}}$ denotes the expected value of the distance between the new node and the node $i$.When the node $j$ add in the system. For large $t N(t)=m_{0}+t \approx t$;

$\frac{\partial k_{i}}{\partial t}=\frac{k_{i} E_{i}\left(d_{i j}\right)^{-1}}{2(1-p) \bar{E} t \overline{\left(d_{i j}\right)^{-1}}}-2 m p \prod \prod^{*} k_{i}$

Then we can get:

$\frac{\partial k_{i}}{\partial t}=\frac{k_{i}}{2(1-p) t}\left(\frac{\left(d_{i j}\right)^{-1}}{\left(d_{i j}\right)^{-1}} \frac{E_{i}}{\bar{E}}-p \frac{d_{i j \max }}{d_{i j \max }} \frac{\bar{E}}{E_{i}}\right)$

It is obvious that, at every time step $t$, when $p=1$, the network cannot grow. Then we consider two cases in the above proposed evolving network model: $p=0$, and $0<p<1$.

In the first case $(p=0)$ :

In this case, the number of link and node is increasing without that of is decline in the evolving process. For large $t$ :

$\frac{\partial k_{i}}{\partial t} \approx \frac{E_{i} k_{i}}{2 \bar{E} t} \frac{\left(d_{i j}\right)^{-1}}{\left(d_{i j}\right)^{-1}}$

With the initial condition $k_{i}\left(t_{i}\right)=m$, then we can get: 
$k_{i}(t)=m \frac{E_{i}}{2 \bar{E}} \frac{\left(d_{i j}\right)^{-1}}{\left(d_{i j}\right)^{-1}}\left(\frac{t}{t_{i}}\right)^{1 / 2}$

Assume $t \rightarrow \infty$, the degree distribution $P(K)$ is as follow:

$P\left(k_{i}(t)<k\right)=p\left(t_{i}>\left(\frac{m E_{i}}{2 \bar{E}} \frac{\left(d_{i j}\right)^{-1}}{\left(d_{i j}\right)^{-1}}\right)^{2} \frac{t}{k^{2}}\right)$

Assuming that we add the node to the network at equal time intervals in evolving process for WSNs, the probability density at the time $t_{i}$ is $p\left(t_{i}\right)=\frac{1}{m_{0}+t}$.Then, we get:

$$
P\left(k_{i}(t)<k\right)=1-\frac{1}{m_{0}+t}\left(\frac{m E_{i}}{2 \bar{E}} \frac{\left(d_{i j}\right)^{-1}}{\left(d_{i j}\right)^{-1}}\right)^{2} \frac{t}{k^{2}}
$$

(1)When the node distribution model is stationary, the probability density distribution function of node energy is:

$$
\begin{aligned}
P\left(k_{E}\right) & =\frac{\partial p\left(k_{i}(t)<k\right)}{\partial k}=\frac{2}{m_{0}+t}\left(\frac{m E_{i}}{2 \bar{E}} \frac{\left(d_{i j}\right)^{-1}}{\left(d_{i j}\right)^{-1}}\right)^{2} \frac{t}{k^{3}} \\
P(k) & =\int_{E \min }^{E \max } \rho(E) P\left(k_{E}\right) d E=\int_{E \min }^{E \max } \rho(E) \frac{2}{m_{0}+t}\left(\frac{m E_{i}}{2 \bar{E}} \frac{\left(d_{i j}\right)^{-1}}{\left(d_{i j}\right)^{-1}}\right)^{2} \frac{t}{k^{3}} d E \\
= & \int_{E \min }^{E \max } \rho(E) \frac{2}{m_{0}+t}\left(\frac{m E_{i}}{2 \bar{E}} \frac{\left(d_{i j}\right)^{-1}}{\left(d_{i j}\right)^{-1}}\right)^{2} \frac{1}{k^{3}} d E, t \rightarrow \infty
\end{aligned}
$$

Where $\rho(E)$ denotes the probability density distribution of node energy in the whole network, $E$ max denotes the maximum value of the node energy; $E$ min denotes the minimum value of the node energy. Therefore, under the same node distribution model, $P(k) \propto \alpha k^{-3}$, $\alpha=\int_{E \min }^{E \max } \rho(E) \frac{2}{m_{0}+t}\left(\frac{m E_{i}}{2 \bar{E}} \frac{\left(d_{i j}\right)^{-1}}{\left(d_{i j}\right)^{-1}}\right)^{2} d E$.It is obvious that $P(K)$ follows the same power law as the BA scale-free model.

(2)When the probability density distribution of node energy in the whole network which is stationary, the probability density distribution function of distance between nodes is:

$$
\begin{aligned}
& P\left(k_{d}\right)=\frac{\partial p\left(k_{i}(t)<k\right)}{\partial k}=\frac{2}{m_{0}+t}\left(\frac{m E_{i}}{2 \bar{E}} \frac{\left(d_{i j}\right)^{-1}}{\left(d_{i j}\right)^{-1}}\right)^{2} \frac{t}{k^{3}} \\
& P(k)=\int_{D \min }^{D \max } \rho(D) P\left(k_{D}\right) d D=\int_{D \min }^{D \max } \rho(D) \frac{2}{m_{0}+t}\left(\frac{m E_{i}}{2 \bar{E}} \frac{\left(d_{i j}\right)^{-1}}{\left(d_{i j}\right)^{-1}}\right)^{2} \frac{t}{k^{3}} d D \\
& =\int_{D \min }^{D \max } \rho(D) \frac{2}{m_{0}+t}\left(\frac{m E_{i}}{2 \bar{E}} \frac{\left(d_{i j}\right)^{-1}}{\left(d_{i j}\right)^{-1}}\right)^{2} \frac{1}{k^{3}} d D, t \rightarrow \infty
\end{aligned}
$$

Where $\rho(D)$ denotes the probability density distribution of the distance between nodes in the whole network. $D_{\max }$ denotes the maximum value of the distance between nodes. $D_{\min }$ denotes the minimum value of the distance between nodes;

In the second case $(0<p<1)$ :

In this case, links and nodes in the evolving network model are not monotonously growing. Instead, links and nodes can be added in some occasion and removed in other case. We rewrite as follow:

$$
\begin{aligned}
& \frac{\partial k_{i}}{\partial t}=\frac{k_{i}}{2(1-p) t}\left(\frac{\left(d_{i j}\right)^{-1}}{\left(d_{i j}\right)^{-1}} \frac{E_{i}}{\bar{E}}-p \frac{d_{i j \max }}{d_{i j \max }} \frac{\bar{E}}{E_{i}}\right) \\
& \alpha=\frac{\left(d_{i j}\right)^{-1}}{\left(d_{i j}\right)^{-1}} \frac{E_{i}}{\bar{E}}-p \frac{d_{i j \max }}{\overline{d_{i j \max }}} \frac{\bar{E}}{E_{i}}
\end{aligned}
$$

With the initial condition $k_{i}\left(t_{i}\right)=m$, then we can get: 
$k_{i}(t)=\left(\frac{t}{t_{i}}\right)^{\frac{\alpha}{2(1-p)}}$

Form (3.16), we can get:

$$
P\left(k_{i}(t)<k\right)=P\left(t_{i}>t^{*} k^{\frac{-2(1-p)}{\alpha}}\right)
$$

Assuming that we add the node to the network at equal time intervals in evolving process for WSNs, the probability density at the time $t_{i}$ is $p\left(t_{i}\right)=\frac{1}{m_{0}+t}$.Then, we get:

$$
P\left(k_{i}(t)<k\right)=1-\frac{t}{m_{o}+t} k^{\frac{-2(1-p)}{\alpha}}
$$

(1)When the node distribution model is stationary, the probability density distribution function of node energy is:

$$
\begin{aligned}
& p\left(k_{E}\right)=\frac{\partial p\left(k_{i}(t)<k\right)}{\partial k}=2 \frac{(1-P)}{\alpha} \frac{t}{m_{0}+t} k^{-\left(\frac{2(1-P)}{\alpha}+1\right)} \\
& P(k)=\int_{E \min }^{E \max } \rho(E) P\left(k_{E}\right) d E=\int_{E \min }^{E \max } \rho(E) 2 \frac{(1-P)}{\alpha} \frac{t}{m_{0}+t} k^{-\left(\frac{2(1-P)}{\alpha}+1\right)} d E,
\end{aligned}
$$

The definition of $\rho(E), E_{\max }$ and $E_{\min }$ is same as ( 3.11$)$.

(2)When the probability density distribution of node energy in the whole network which is stationary, the probability density distribution function of distance between nodes is:

$$
\begin{aligned}
& p\left(k_{d}\right)=\frac{\partial p\left(k_{i}(t)<k\right)}{\partial k}=2 \frac{(1-P)}{\alpha} \frac{t}{m_{0}+t} k^{-\left(\frac{2(1-P)}{\alpha}+1\right)} \\
& P(k)=\int_{D \text { min }}^{D \max } \rho(D) P\left(k_{D}\right) d D=\int_{D \text { min }}^{D \max } \rho(D) 2 \frac{(1-P)}{\alpha} \frac{t}{m_{0}+t} k^{-\left(\frac{2(1-P)}{\alpha}+1\right)} d D,
\end{aligned}
$$

The definition of $\rho(D), D_{\max }, D_{\min }$ is same as (3.13).

\section{The Simulation and Analysis}

4.1 The Parameter setting and the Analysis of the degree distribution $P(K)$

In this paper, we want to compare with these two conditions as: different node energy models and different node distribution models, under these two conditions, how the network's important parameter change. Simulation tool is MATLAB 2011b.

The parameter setting is as follows:

1. $N=1000, N$ denotes the number of node in the whole network.

2. $m_{0}=4, m_{0}$ denotes the number of node in the initial whole network.

3. $m=4, m$ denotes that when adding a new node to the network, the network will add $m$ links.

4. $p=0.5, p$ denotes the deletion probability.

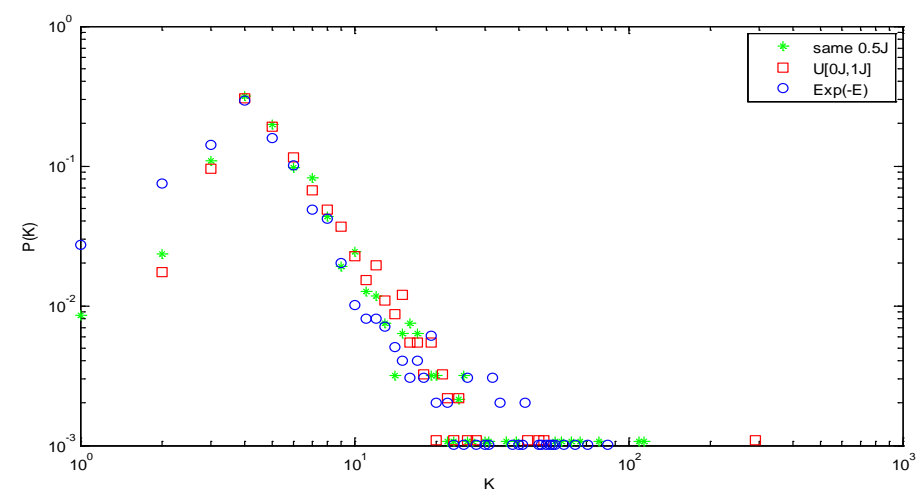

Figure 1: The degree distribution $P(K)$ obtained by simulations as under random location distribution model, with three kinds of $\rho(E)$. 
In Figure 1, we can see that the degree distributions $P(K)$ are power law as the same as B-A model. Because random position distribution model is irregular position distribution model. If the degree distribution $P(K)$ obtained by simulations in the condition that random location distribution model is power law. Then the degree distribution $P(K)$ obtained by simulations in the condition that other three kinds of location distribution model is also power law.

We consider three kinds of the node energy distribution $\rho(E)$ in the whole network within the interval $[0 j, 1 j]$ :the same node energy with value $0.5 j$ with $\sigma=0$;(2)uniform distribution ( $U[0 j, 1 j]$ )with $\sigma=1 / 12$;(3)exponential distribution ( $\exp (-\mathrm{E})$ )with $\sigma=1$, where $\sigma$ is the standard deviation used to indicate the node energy heterogeneity. And we also consider four kinds of the node distribution model. We set a $1000 \mathrm{~m} * 1000 \mathrm{~m}$ square area. Nodes of distribution model follows the following four distribution respectively :(1) Random distribution; nodes of ordinate and abscissa assume irregular state. (2) Uniform distribution; ( $[0,1000])$ nodes uniformly distribute in the square area. (3) Normal distribution; (norm $(500,1)$ ) nodes normally distribute in the square area. (4) Exponential distribution; ( $\exp (1,1000))$ with $\sigma=1$.

\subsection{Clustering Coefficient}

We investigate the effect of node energy distribution on network's cluster coefficient, which quantifies the extent to which nodes adjacent to a given node are linked. It is defined as follows:

$C=\frac{1}{N} \sum_{i} C_{i}=\frac{1}{N} \sum_{i} \frac{E_{i}}{k_{i}\left(k_{i}-1\right) / 2}$.

$N$ denotes the number nodes in the whole network; $E_{i}$ denote the number of edges among the neighbor nodes of a selected node $i$ with degree $k_{i}$ in the network.

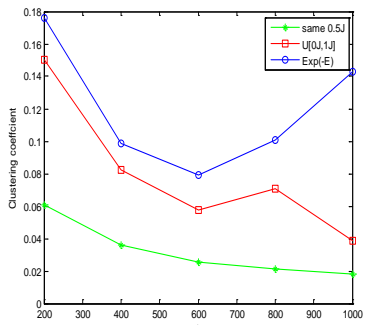

Figure2:Uniform

distribution of

location model

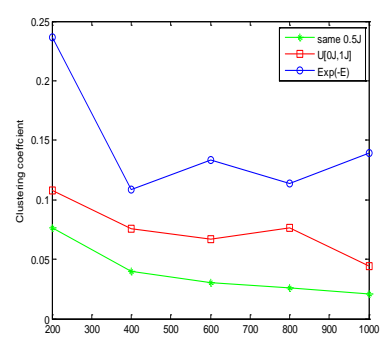

Figure3:Random distribution of location model

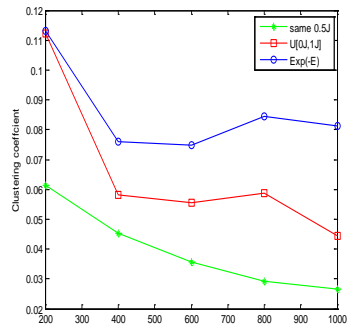

Figure4:Exponential distribution of location model

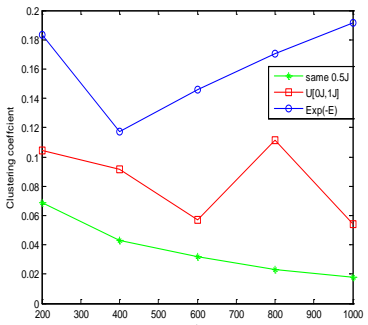

Figure4:Normal distribution of location model

Comparing with Figure2, Figure3, Figure4 and Figure5, we can see that the value of $C$ is high when $\rho(E)$ is exponential distribution. But when node energy is same, $C$ keeps very small value. The result illustrate that nodes energy is more heterogeneity, the network is more clustering.

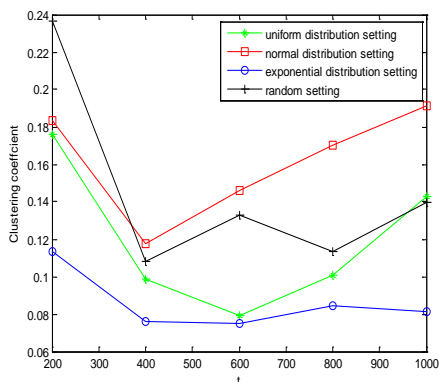

Figure6:Exponential distribution energy model

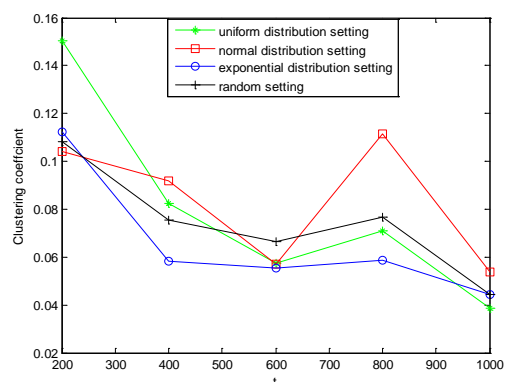

Figure7: Uniform distribution energy mode

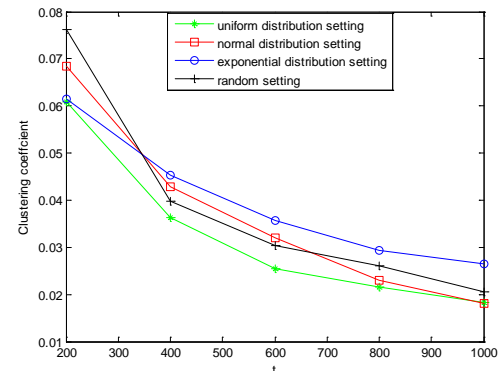

Figure8: Same energy model 
Comparing with Figure6, Figure7 and Figure8, we can see that when nodes energy are heterogenous, $C$ keeps high value with normal distribution setting model. But $C$ have small value with exponential distribution setting model. When nodes energy is same, $C$ keeps very low value with these four nodes setting model.

\subsection{Average Shortest Path Length}

In WSN, the senor nodes transmit data by multihopes method. The average path length $L$ is defined to be the average length of the shortest paths between any two nodes in the network. $L$ characterize the ability of two nodes to communicate with each other, the smaller $L$ mean fewer hops and less energy consumption for data processing. Its formulation can be defined as:

$L=\frac{1}{N(N-1)} \sum_{i \neq j} d_{i j}$.

$N$ denotes the number of node in the whole network; $d_{i j}$ denotes the length of the shortest path between node $i$ and node $j$.

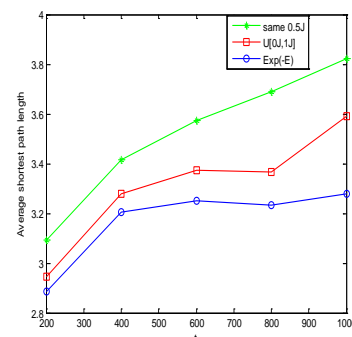

Figure 9:Exponential

Distribution setting model

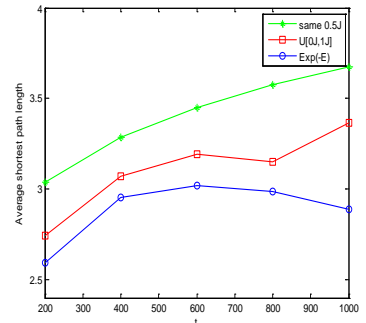

Figure10: Normal

Distribution setting model

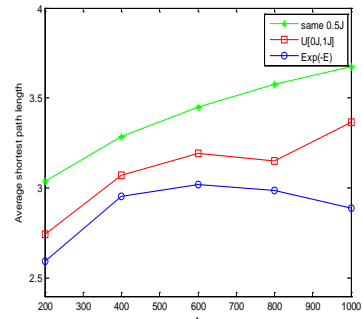

Figure11: Uniform

Distribution setting model

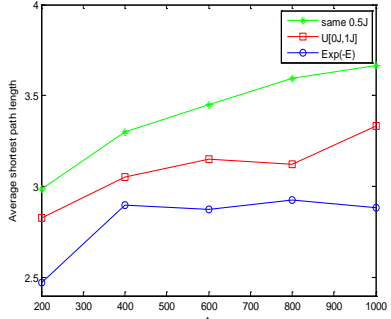

Figure12: Random

Distribution setting model

Comparing with Figure9, Figure10, Figure 11 and Figure 12. we can see that the value of $L$ is small when $\rho(E)$ is exponential distribution. But when node energy is same, $L$ keeps very high value. And we find that $L$ increases with the network size increasing. $L$ keeps a value of 2.7, when the number of node in the whole network are 400 with $\rho(E)$ is exponential distribution. And the number of node in the whole network increase, $L$ keeps the value of 2.7. The result illustrate that nodes in energy is more inhomogeneous, the sensor communicate with others more efficiently.

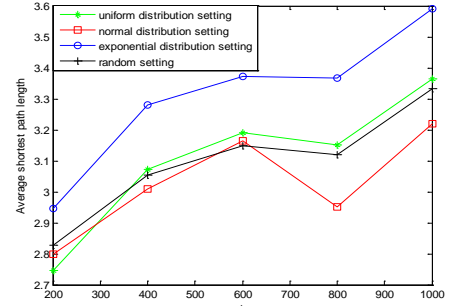

Figure 13: Uniform distribution energv model

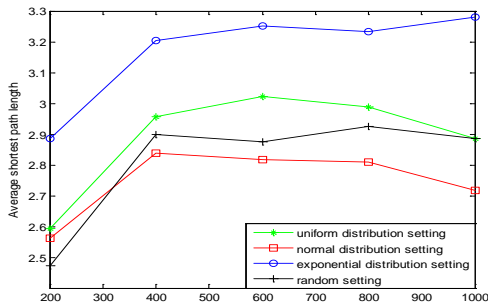

Figure 13:Same energy model

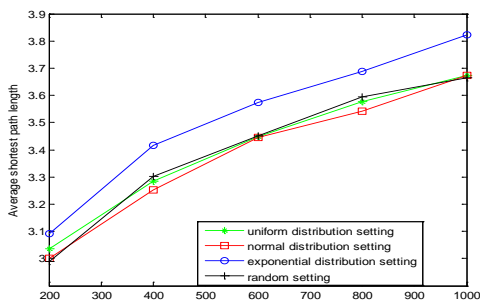

Figure 13: Exponential distribution energy model

Comparing with Figure13, Figure14 and Figure 15. We can see that with the three kinds of energy distribution models, we can find that when the setting model is exponential distribution setting model, $L$ keeps a high value. And when the setting model is normal distribution setting model, $L$ keeps a small value.

\section{Conclusion}

In this paper, we propose a novel topology model for wireless sensor networks. This model 
combined location-aware mechanism with energy-aware mechanism. By using mean-field approach, the degree distribution $P(K)$ is power law. We also use MATLAB software to get the results derived in this paper. Node energy distribution and node location distribution have a weak effect on the degree distribution $P(K)$ but these have much effect on the network internal topological characterizations. The node which has more energy will have more degrees for balancing energy consumption, and the model exhibits that the network is high clustering for the sensor network system in which node energy is more heterogeneous and node location is more centralized. Then, we find that, when node energy distribution is more heterogeneous and node location is more centralized, the network enjoys better performance in energy efficiency for transmitting data.

This model provides some useful for constructing WSNS. When we want to get a best performance of a WSN, we can choose normal distribution setting model and set about 800 nodes with heterogeneous energy in the field which should be monitored.

\section{Acknowledgements}

This work was supported by the National Natural Science Foundation of China (grant No.60963026, No.61163061), the 13'th Kunming and YMU Youth Academic and Technical Preparatory Talents Project and 2014's Teaching Reform Project of Yunnan Minzu University(Grant No.15002050).

The corresponding author is Fan Jing Ph.D.Party Secretary \&Professor School of Electric and Information EngineeringYunnan MinZu University Yuhua Campus, Chenggong University City Kunming, Yunnan 650500, P.R.China.

\section{References}

[1] R. Albert, H. Jeong and A.-L. Barabasi. Error and attack tolerance of complex networks. Nature, 2000; 406: 378-382.

[2] Faragó A. Network topology models for multihop wireless networks[J]. ISRN Communications and Networking, 2012, 2012: 22.

[3] Mahesar A W, Malik M, Abid H, et al. Calculus and its applications in scale-free networks [C]// Information and Communication Technology for The Muslim World (ICT4M), 2014 The 5th International Conference on. IEEE, 2014: 1-6.

[4] Li-Jun C, Dao-xu C, Li X, et al. Evolution of wireless sensor network [C]//Wireless Communications and Networking Conference, 2007. WCNC 2007. IEEE. IEEE, 2007:3003-3007. [5] Zhu H, Luo H, Peng H, et al. Complex networks-based energy-efficient evolution model for wireless sensor networks[J]. Chaos, Solitons \& Fractals, 2009, 41(4): 1828-1835.

[6] Zheng G, Liu S, Qi X. Scale-free topology evolution for wireless sensor networks with reconstruction mechanism[J]. Computers \& Electrical Engineering, 2012, 38(3): 643-651.

[7] Luo X, Yu H, Wang X. Energy-aware topology evolution model with link and node deletion in wireless sensor networks[J]. Mathematical problems in engineering, 2011, 2012.

[8] Ye D, Zhang M. A Self-Adaptive Strategy for Evolution of Cooperation in Distributed Networks [J]. Computers, IEEE Transactions on, 2015, 64(4): 899-911. 